\title{
A MODEL TO EVALUATE ROAD TRAFFIC SAFETY IN SAFE
} COMMUNITIES (SC)

doi:10.1136/injuryprev-2012-040590w.17

${ }^{1} \mathrm{SM}$ Hashemi, ${ }^{2} \mathrm{M}$ Zangi, ${ }^{2} \mathrm{R}$ Mohammadi. ${ }^{1}$ Azad University, Tehran, Iran; ${ }^{2}$ Karolinska Institutet, Stockholm, Sweden

Background Traffic is still the leading cause of death and disability in all around world and indeed SC Evaluation in terms of traffic safety is crucial.

Aims To set up a comprehensive model to assess traffic safety in SCs.

Methods Traffic safety parts of SC reports and related scientific articles in Pubmed and Embase were reviewed to find evaluation methods of traffic safety.

Results Three main methods of traffic safety evaluation have been developed as follows: Firstly, process evaluation, in which, focus group discussion among members of traffic safety work team is recommended. Secondly, impact evaluation, in which, quasi experimental study with emphasis on usage of safety equipment such as Seat belts, child restraints, bicycle helmets, motorcycle helmets by drivers and passengers is best work to be carried out at designation and re-designation step between SC and control community. Finally, outcome evaluation, in which, in another quasi experimental study, incidence, severity and burden of traffic accidents is estimated and compared between SC and control community. Besides WHO and CDC questionnaires, delphi technique is an useful alternative to prepare questionnaires.

Contribution to the Field The quite large number of traffic accidents in the world justifies the importance of designing a model to evaluate SC interventions regarding road traffic safety. 